\section{Variability in Rehabilitation Protocols after Superior Labrum Anterior Posterior Surgical Repair}

Christina A. Hermanns ${ }^{1}$, Reed G. Coda', Sana Cheema', Matthew L. Vopat, M.D. ${ }^{2}$, Armin Tarakemeh ${ }^{3}$, Kyle Veazey, D.P.T., , John P. Schroeppel, M.D. ${ }^{3}$, Scott Mullen, M.D. ${ }^{3}$, Bryan G. Vopat, M.D. ${ }^{3}$ ${ }^{1}$ University of Kansas School of Medicine, Kansas City, KS ${ }^{2}$ University of Kansas School of Medicine-Wichita, Wichita, KS, Department of Orthopaedics

${ }^{3}$ University of Kansas Medical Center, Kansas City, KS, Department of Orthopedic Surgery

${ }^{4}$ University of Kansas Medical Center, Kansas City, KS, Department of Physical Therapy

Received March 22, 2021; Accepted for publication. June 4, 2021; Published online Oct. 14, 2021 https: /doi.org/10.17161/ljm.voll4.15286

\section{ABSTRACT}

Introduction. Rehabilitation after a superior labral anterior posterior (SLAP) repair is an important aspect of patient outcomes; however, no standardized rehabilitation protocol has been defined. The purpose of this paper is to assess the variability of rehabilitation after a SLAP repair to understand the need for standardization to improve patient outcomes.

Methods. Protocols for SLAP repairs were collected through a search for Academic Orthopedic Programs and a general Google search using the terms "[Program Name (if applicable)] SLAP Repair Rehab Protocol". Protocols were compared by sling, range of motion (ROM), physical therapy, return to sport (RTS), return to throwing, and biceps engagement and tenodesis recommendations. Protocols for non-operative or generalized shoulders were excluded.

Results. Sixty protocols were included. A total of $61.7 \%$ (37/60) recommended a sling for four to six weeks and 90\% (54/60) included a full ROM recommendation, but time was variable. There were different exercises recommended, but pendulum swings were recommended by $53 \%$ (32/60), submaximal isometrics by $55 \%$ (33/60), and scapular strengthening by $65 \%$ (39/60). Of the sixty protocols, $33 \%$ (20/60) recommended return to sports in 24 weeks and $38.3 \%$ (23/60) recommended allowing throwing in 16 weeks.

Conclusions. There was variability in protocols for SLAP repair, especially time until full ROM, RTS, and biceps strengthening. Time in sling and scapular strengthening were the least variable. A lack of specificity within protocols in what return to throwing meant for functional ability made it difficult to compare protocols. Considering the large number of orthopedic programs, a relatively small number had published protocols. Further studies are needed to evaluate a standardized post-operative rehabilitation for SLAP repairs to improve outcomes.

\section{Kans J Med 2021;14:243-248}

\section{INTRODUCTION}

Superior labral anterior to posterior (SLAP) tears are a major cause of shoulder dysfunction, often found in overhead and throwing athletes. ${ }^{1}$ They occur due to tensile overload when eccentric bicep contractions lift the bicep tendon off of the glenoid insertion which leads to labral injuries. ${ }^{2}$ Traditionally, the management of SLAP lesions begins with non-operative treatment for three to six months, which can include

\section{KANSAS JOURNAL of MEDICINE}

supervised rehabilitation, nonsteroidal anti-inflammatory drugs, and intra-articular corticosteroid injections to improve the function and pain in the joint. ${ }^{3}$ If conservative management fails to regain the patient's full range of motion (ROM) or return to the desired level of activity, surgical intervention may be considered.

Surgical treatment varies depending on the type of SLAP tear and the repair can be done through a variety of techniques. SLAP repairs have shown variable rates of success, ranging from some studies citing good to excellent outcomes, while others report less favorable results. ${ }^{4-10}$ A systematic review by Gorantla et al. ${ }^{11}$ found good to excellent results ranged from 40 to $94 \%$ of patients in studies, return to previous activity level ranged from 20 to $94 \%$, and return of overhead athletes to previous level ranged from 22 to $64 \%$. If significant biceps tendon pathology is also present, biceps tenodesis also may be performed at the same time as the SLAP repair. ${ }^{3}$ The presence of concomitant surgery such as this would be expected to lead to changes in rehabilitation.

Suggestions for rehabilitation after surgical repair of a SLAP lesion have been outlined in the literature, but has never been reviewed in protocols used in practice. Manske et al. ${ }^{12}$ described the rehabilitation in five phases based on a literature review: protective phase from week zero to six, moderate protection phase from week 7 to 12 , minimum protection phase from week 13 to 20 , advanced strengthening phase from week 21 to 26 , and return to activity from months six to nine. However, as stated previously, these are just suggestions and a study has yet to find a standardized agreement for post-operative rehabilitation of SLAP repairs.

The glenohumeral joint is one of the most mobile in the body; its stability comes from interactions between the joint, muscles, tendons, capsule, osseous configuration, and the glenoid labrum. ${ }^{12}$ Therefore, successful outcomes depend directly on a balance between mobilizing the shoulder while strengthening the rotator cuff and scapulothoracic musculature. Thus, after a SLAP repair, the post-operative rehabilitation of the surrounding musculature is essential to the recovery of the functional joint. However, in a systematic review to analyze the current treatment practices for SLAP lesions, Kibler et al. ${ }^{1}$ found that there was a severe lack in published guidelines on rehabilitation recommendations. Therefore, the purpose of this study is to analyze the variability of all published rehabilitation protocols for post-operative care after a SLAP repair. If SLAP repair protocols can be gathered and analyzed, then general conclusions and trends of SLAP rehabilitation can be summarized and areas lacking consensus can be identified.

\section{METHODS}

A list of all orthopedic academic residency programs was obtained using the Fellowship and Residency Electronic Interactive Database Access (FREIDA) program search tool through the American Medical Association. These programs framed the Google search for "[Program Name] SLAP repair rehab protocol” to find all published protocols from academic orthopedic programs within the U.S. Any private practice protocols found during this search also were collected. An additional general search for "SLAP repair rehab protocol" was conducted to 
KANSAS JOURNAL of MEDICINE

REHABILITATION PROTOCOLS AFTER SLAP REPAIR continued.

search for any additional protocols. Due to this being a review of currently published post-operative protocols, an Institutional Review Board (IRB) approval was not needed.

Non-operative care, generalized shoulder rehabilitation, and nonEnglish protocols were excluded. Records that were excluded due to being too general meant that the protocol covered broad rehabilitation for most procedures done to the shoulder (e.g., rotator cuff repair, Bankart repair, and total shoulder arthroplasty). A flow chart of this process is shown in Figure 1.

Each protocol was compared for variability in the inclusion, exclusion, and timing of sling immobilization, ROM guidelines, physical therapy exercises, return to throwing, return to sport (RTS), biceps strengthening, and if the protocol included additional guidelines for the presence of concomitant biceps tenodesis. For specific physical therapy exercises, only exercises that were present in more than $10 \%$ of protocols were included in the results of this paper.
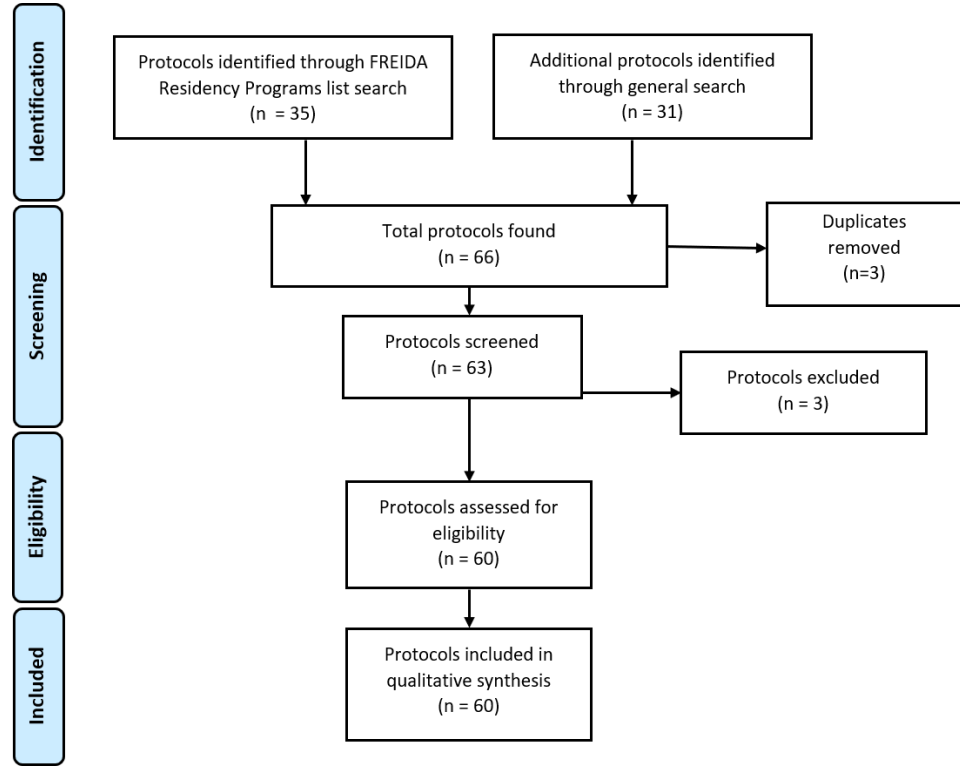

Figure l. Flow diagram of methods of data collection used in this study.

\section{RESULTS}

A total of 60 protocols was included in this study. There were 27 U.S. academic residency programs that published SLAP rehabilitation protocols (14.7\% of the 183 academic programs). Two academic programs had different published protocols from different physicians within their system; both were included in this study, making the total academic protocols used 29. There were 31 private practice protocols found and included in the study. A list of protocols included can be found in the Appendix.

Immobilization. A total of $97 \%$ (58/60) of protocols mentioned wearing a sling post-operatively (Figure 2). The majority of protocols $(61.7 \% ; 37 / 60)$ recommended that patients wear the sling until the four to six week timeframe, while $15 \%$ (9/60) recommended wearing the sling for two to four weeks, $13 \%(8 / 60)$ recommended for six or more weeks, and 6\% (4/60) per surgeon's approval. However, 3\% (2/60) did not specify the use of a sling post-operatively.

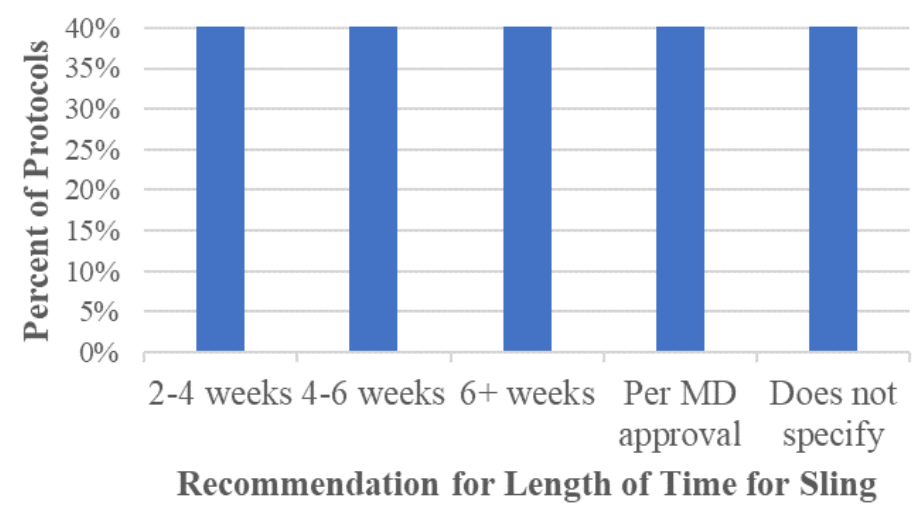

Figure 2. The length of time a protocol recommended wearing a sling and the number of protocols that recommended that timeframe.

Range of Motion (ROM). A total of 83\% (50/60) of the protocols specified ROM recommendations starting at day or week zero, meaning immediately post-operatively (Figure 3). Sixty percent $(36 / 60)$ of the protocols allowed passive ROM (PROM) immediately after surgery, while $8.3 \%$ (5/60) recommended no ROM, and $15 \%$ (9/60) allowed active assisted ROM (AAROM) of the shoulder. However, $16.7 \%$ (10/60) of the protocols did not specify post-operative ROM. Most patients were immobilized in a sling while instructed to do certain motion exercises such as pendulums, elbow, and wrist exercises which were counted as allowing passive or AAROM.

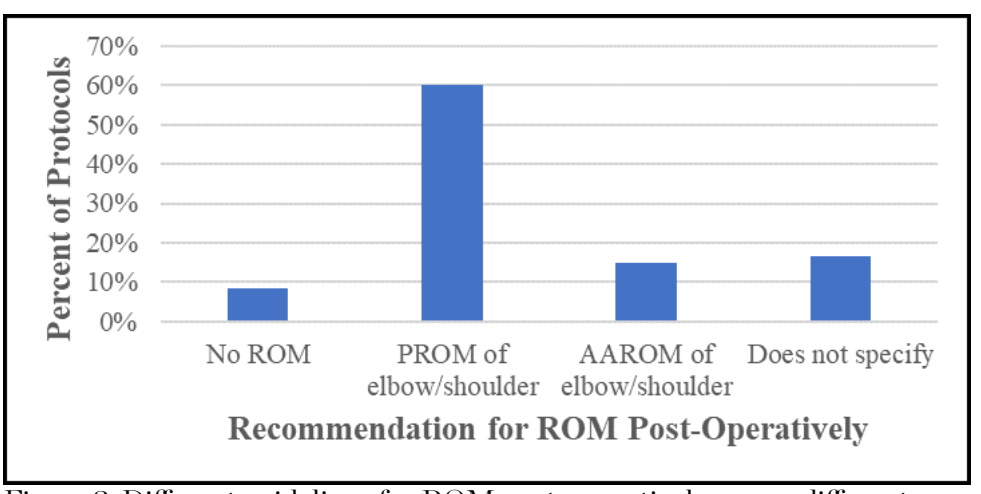

Figure 3. Different guidelines for ROM post-operatively across different protocols. (PROM: passive range of motion; AAROM: active assisted range of motion)

A total of $88.3 \%$ of protocols $(53 / 60)$ included a guideline for full ROM at a certain week (Figure 4). One percent of protocols (1/60) left full ROM up to physician clearance, and 10\% (6/60) did not specify full ROM. Of the protocols that specified agoal for full ROM, $18.3 \%$ (11/60) aimed for before eight weeks, $20 \%$ (12/60) for eight to ten weeks, $18.3 \%$ (11/60) for 10 to 12 weeks, $23 \%$ (14/60) for 12 to 14 weeks, $3 \%(2 / 60)$ for 14 to 16 weeks, and $5 \%(3 / 60)$ for $16+$ weeks.

Exercises. A total of 65\% (39/60) of protocols recommended scapular strengthening movements. Fifty-five percent (33/60) of protocols recommended using submaximal isometric exercises to strengthen the upper extremity. Fifty-three percent (32/60) of protocols recommended using pendulum swings, most of which recommended these begin relatively soon after surgery. All exercises that were mentioned in more than $10 \%$ of protocols can be seen in Figures 5 and 6. 


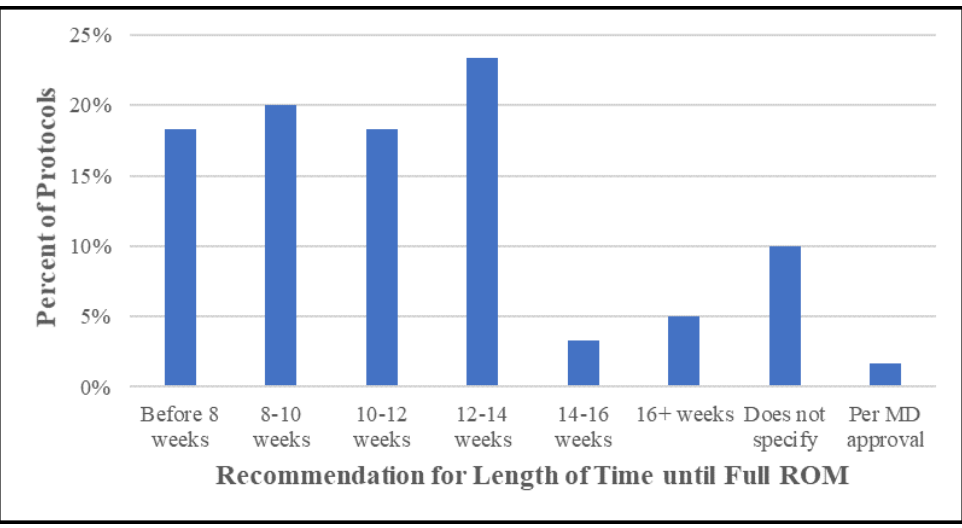

Figure 4. Time when full ROM of the shoulder is allowed and the percentage of protocols that recommended allowing full ROM at that time.

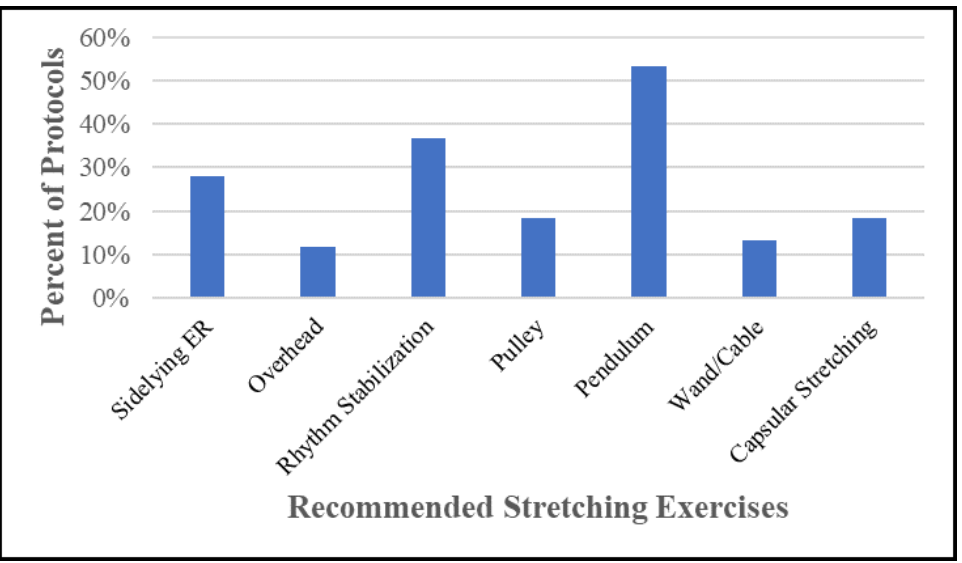

Figure 5. Stretching exercise recommendations to improve ROM and percentage of protocols that recommended that exercise. Only exercises that were present in more than $10 \%$ of protocols were included in this graph.

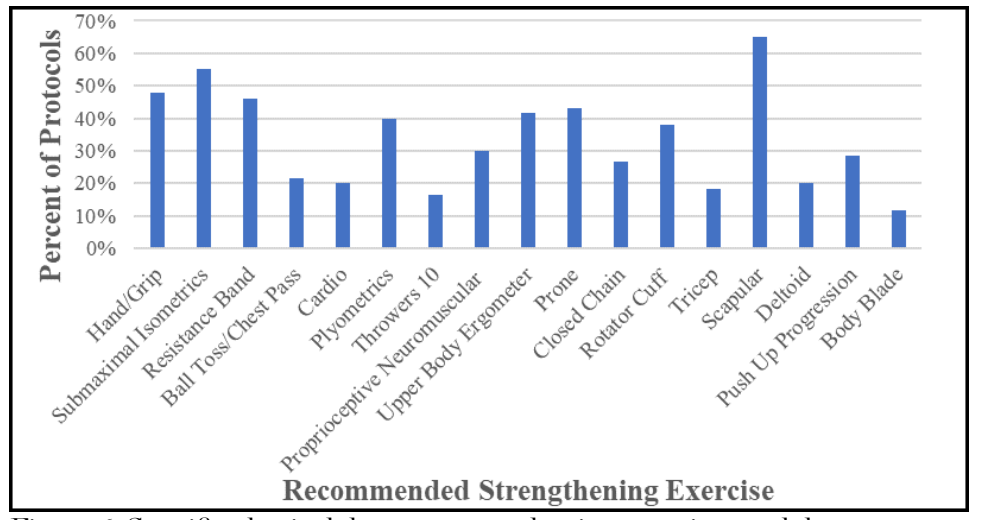

Figure 6. Specific physical therapy strengthening exercises and the percentage of protocols that included them. Only exercises that were present in more than $10 \%$ of protocols were included in this graph.

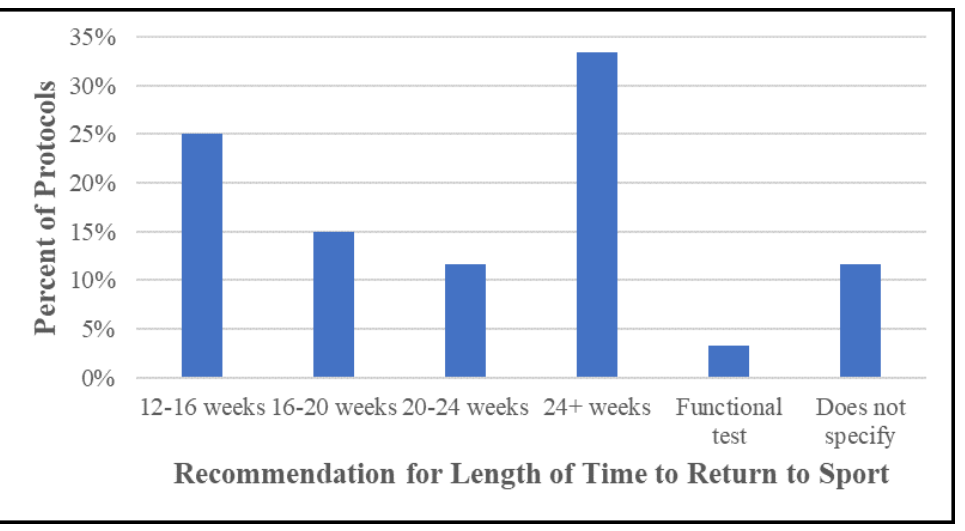

Figure 7. Percentage of protocols that included time of RTS post-operatively.

\section{KANSAS JOURNAL of MEDICINE}

REHABILITATION PROTOCOLS AFTER SLAP REPAIR continued.

Return to Sport (RTS) and Throwing. A total of $88.3 \%$ (53/60) of protocols included RTS (Figure 7). The largest group of protocols, at $33 \%(20 / 60)$, recommended RTS at $24+$ weeks, while $25 \%$ (15/60) recommended 12 to 16 weeks, $15 \%$ (9/60) recommended 16 to 20 weeks, and $11.6 \%$ (7/60) recommended 20 to 24 weeks. Only $3 \%$ $(2 / 60)$ required functional testing, and $11.6 \%(7 / 60)$ did not specify time until RTS.

Of the 60 protocols, $68.3 \%$ (41/60) included return to throwing (Figure 8). Thirty-eight percent (23/60) of protocols allowed some sort of throwing motion to begin around 16 to 20 weeks. Ten percent $(6 / 60)$ allowed return to throwing at 12 to 16 weeks, $10 \%(6 / 60)$ at 20 to 24 weeks, $8 \%(5 / 60)$ at $24+$ weeks, $1.6 \%(1 / 60)$ per MD approval, and $31.6 \%(19 / 60)$ did not specify.

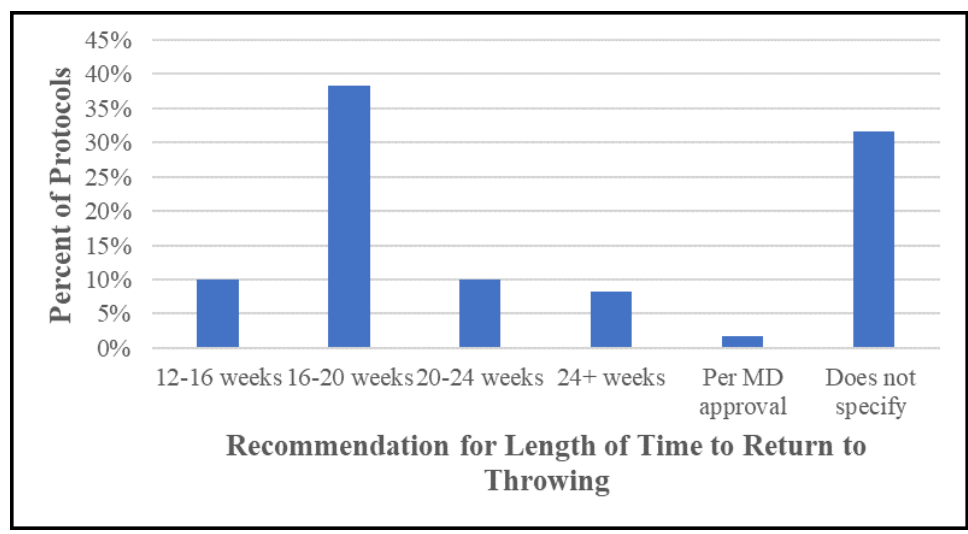

Figure 8. Percentage of protocols that included the initiation of throwing motions at different timeframes post-operatively.

Biceps Engagement and Tenodesis. A total of 36.7\% (22/60) of the protocols specified a guideline for restraining biceps engagement until a certain time point after surgery to protect the healing process (Figure 9). Of those, 1.6\% (1/60) allowed biceps engagement before six weeks, $18.3 \%$ (11/60) at six to eight weeks, $5 \%(3 / 60)$ at eight to ten weeks, and $11.6 \%$ (7/60) at 10 to 12 weeks. Sixty-three percent (38/60) of protocols did not specify a biceps engagement or strengthening timeframe.

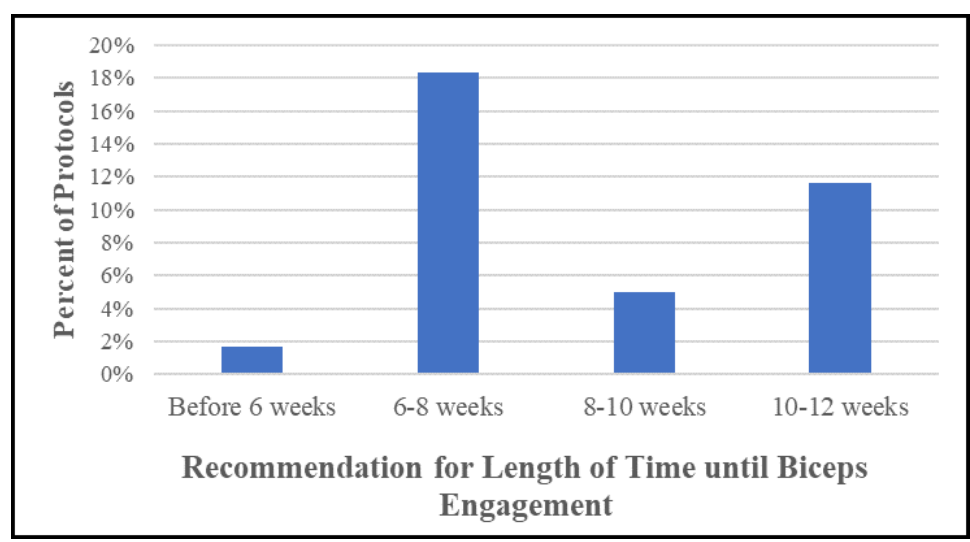

Figure 9. Percentage of protocols that recommend allowing bicep engagement at different time points post-operatively. 
KANSAS JOURNAL of MEDICINE REHABILITATION PROTOCOLS AFTER SLAP REPAIR continued.

Only 6\% (4/60) of protocols specified the presence of or differences in rehabilitation due to the addition of concomitant biceps tenodesis with a SLAP repair. Two of the four protocols did not change rehabilitation with the addition of biceps tenodesis. The other two added on the following precautions if biceps tenodesis also was performed: no resisted elbow flexion for six weeks or eight weeks depending on protocol, no resisted shoulder flexion for eight weeks, no lifting anything over one or two pounds for eight weeks, and avoiding 90/90 stretching with previous instability for six weeks.

\section{DISCUSSION}

This study illustrated a high variability across SLAP repair post-operative protocols, especially in terms of time to full ROM, rehabilitation exercises, RTS, and biceps engagement. There was also a lack of published protocols from most U.S. academic orthopedic programs, although a sizable number of protocols were found to be analyzed. There were some general trends in protocols, including most (77\%, $46 / 60)$ protocols recommending four weeks of immobilization.

Most protocols $(61.6 \%, 37 / 60)$ recommended wearing a sling for four to six weeks, but allowed some ROM after surgery. All protocols in which ROM was specified recommended immediate motion after surgery, although the recommendation on passive versus active assisted ROM was variable. In terms of time to full ROM, the variability in rehabilitation protocols matched up with a lack of guidelines in the literature. ${ }^{1}$ Eighty percent (48/60) of protocols recommended a goal of full ROM by 12 weeks or sooner. The longest recommended timeframe for full ROM was 12 to 14 weeks with only $23.3 \%$ (14/60) of protocols. Based on the data in this study, if a patient had not achieved full ROM by around 12 weeks, they would be lagging based on protocol standards. However, Michener et al. ${ }^{3}$ suggested that ROM should recover fully after a SLAP repair, but because the course of a patient's increasing motion ability throughout post-operative treatment has not been measured, the time to full ROM recommendations could not be determined. This may help explain the variability in time to full ROM seen across protocols.

The general guideline published by Michener et al. ${ }^{3}$ outlines returning to an interval throwing program at approximately four months (16 weeks) post-operatively, and RTS at around six months (24 weeks) after surgery. These guidelines were created from a literature search of current research on how to optimally manage SLAP tears. The longest recommended RTS timeframe found in protocols was 24 weeks $(33.3 \%, 20 / 60)$ and return to a throwing program was 16 to 20 weeks $(38.3 \%, 23 / 60)$. This was consistent with the literature, but neither group of protocols that recommended these timeframes represented a majority of all protocols (33\% and $38.3 \%$, respectively). The variability in RTS may be due to the vague nature of what RTS meant in terms of activity and movement, as well as individualized rehabilitation programs and differences between patients. Thus, it may be more accurate to specify which sport they return to, such as contact (e.g., football and rugby) versus non-contact sports (e.g., tennis and golf), and their level of participation in that sport (e.g., professional or recreational). However, it would be beneficial to know a general timeline of how long it should take a patient to progress to a level of function that they will be ready to RTS so that a practitioner can have an idea of whether a patient is improving as expected. More studies are needed to evaluate what the exact RTS recommendation should be for each sport. Return to throwing may be similar as some protocols mentioned return to throwing as the beginning of a progression to previous throwing levels instead of an actual return to full throwing. In this study, the results regarding use of functional testing to clear an athlete for sports were limited in protocols. This was consistent with the lacking and limited evidence of upper extremity functional testing, especially in injured populations, in the current litereature. ${ }^{13}$

There is a need for an increased number of published protocols to standardize timelines with literature evidence as well as to detail specific guidelines to ensure that patients recover optimally from a SLAP repair, regardless of where their procedure was performed. Authors of this study acknowledge that SLAP injuries themselves include an array of injuries and each patient's expectations also may vary. Thus, this study illustrated that there is a need for additional studies to clinically look at standardized post-operative protocols for specific patients, sports, and severity of injury.

\section{LIMITATIONS}

This study was limited by the number of published protocols found and the lack of online publications of rehabilitation protocols by many institutions. Regarding categories such as exercise recommendations and ROM, there was a large variety of included recommendations and formatting of rehabilitation protocols, which made it difficult to consolidate all protocols. Many protocols were also vague in specifics of what motion they meant by recommending certain movements and exercises.

\section{CONCLUSIONS}

There was variability in protocols for SLAP repair, especially time until full ROM, RTS, and biceps strengthening. Time in sling and scapular strengthening were the least variable. A lack of specificity within protocols in what return to throwing meant for functional ability made it difficult to compare protocols. Considering the large number of orthopedic programs, a relatively small number had published protocols. Further studies are needed to evaluate a standardized post-operative rehabilitation for SLAP repairs to improve outcomes.

\section{REFERENCES}

${ }^{1}$ Kibler WB, Sciascia A. Current practice for the surgical treatment of SLAP lesions: A systematic review. Arthroscopy 2016; 32(4):669-683. PMID: 26553961.

${ }^{2}$ Beyzadeoglu T, Circi E. Superior labrum anterior posterior lesions and associated injuries: Return to play in elite athletes. Orthop J Sports Med 2015; 3(4):2325967115577359. PMID: 26665050;

${ }^{3}$ Michener LA, Abrams JS, Bliven KCH, et al. National Athletic Trainers' Association Position Statement: Evaluation, management, and outcomes of and return-to-play criteria for overhead athletes with superior labral anterior-posterior injuries. J Athl Train 2018; 53(3):209-229.

PMID: 29624450.

${ }^{4}$ Steinhaus ME, Makhni EC, Lieber AC, et al. Variable reporting of functional outcomes and return to play in superior labrum anterior and posterior tear. J Shoulder Elbow Surg 2016; 25(11):1896-1905. PMID: 27497788. 
5 Denard PJ, Ladermann A, Burkhart SS. Long-term outcome after arthroscopic repair of type II SLAP lesions: Results according to age and workers' compensation status. Arthroscopy 2012; 28(4):45l-457. PMID: 22264832.

${ }^{6}$ Schroder CP, Skare O, Gjengedal E, Uppheim G, Reikeras O, Brox JI. Longterm results after SLAP repair: A 5-year follow-up study of 107 patients with comparison of patients aged over and under 40 years. Arthroscopy 2012; 28(11):1601-1607. PMID: 22608888.

7 Brockmeier SF, Voos JE, Williams RJ 3rd, Altchek DW, Cordasco FA, Allen AA. Outcomes after arthroscopic repair of type-II SLAP lesions. J Bone Joint Surg Am 2009; 91(7):1595-1603. PMID: 19571081.

8 Cohen DB, Coleman S, Drakos MC, et al. Outcomes of isolated type II SLAP lesions treated with arthroscopic fixation using a bioabsorbable tack. Arthroscopy 2006; 22(2):136-142. PMID: 16458798.

${ }^{9}$ Douglas L, Whitaker J, Nyland J, et al. Return to play and performance perceptions of baseball players after isolated SLAP tear repair. Orthop J Sports Med 2019; 7(3):2325967119829486. PMID: 30873424.

${ }_{10}$ Gilliam BD, Douglas L, Fleisig GS, et al. Return to play and outcomes in baseball players after superior labral anterior-posterior repairs. Am J Sports Med 2018; 46(1):109-115. PMID: 28942657.

${ }^{11}$ Gorantla K, Gill C, Wright RW. The outcome of type II SLAP repair: A systematic review. Arthroscopy 2010; 26(4):537-545. PMID: 20362836.

${ }_{12}$ Manske R, Prohaska D. Superior labrum anterior to posterior (SLAP) rehabilitation in the overhead athlete. Phys Ther Sport 2010; 11(4):110-121. PMID: 21055704.

13 Tarara DT, Fogaca LK, Taylor JB, Hegedus EJ. Clinician-friendly physical performance tests in athletes part 3: A systematic review of measurement properties and correlations to injury for tests in the upper extremity. $\mathrm{Br} \mathrm{J}$ Sports Med 2016; 50(9):545-551. PMID: 26701926.

Keywords: shoulder injuries, orthopedic rehabilitation surgery, SLAP tears, range of motion, return to sport
KANSAS JOURNAL of MEDICINE REHABILITATION PROTOCOLSAFTER SLAP REPAIR continued. 


\section{APPENDIX}

\section{List of Protocols}

\section{Type of Institution}

U.S. Academic Orthopedic Program ( $n=29)$

\section{Protocols Obtained From:}

Baylor School of Medicine

Brigham and Women's Hospital

Brown (University Orthopaedics)

Case Medical Center

University of Cincinnati Medical Center

University of Colorado

Columbia University

University of Delaware

Loma Linda

Massachusetts General Hospital Orthopaedics

RUSH - Midwest Orthopaedics

Naval Medical Center

NYU - Dr. Laith Jazrawi

NYU - Dr. Eric Strauss

Ohio State University Wexner Medical Center

Saint Louis University

St. Mary Hospital and Medical Center

UCLA

University of Florida

UNM School of Medicine

Keck School of Medicine of USC

UT Health

University of Wisconsin Sports Medicine

Vanderbilt Sports Medicine

Vanderbilt Sports Medicine Knee and Shoulder Center

VCU Sports Medicine Clinic

University of Virginia Health System

University of Washington Medical Center

William Beaumont Army Medical Center

Private Practice $(\mathrm{n}=31)$

Beaumont Hospital

Summit Sports Medicine

Boston Sports Medicine \& Research Institute

Center Sports Medicine and Orthopedics

Central Texas Sports Medicine and Orthopedics

Chicago Orthopaedics and Sports Medicine

Elite Sports Medicine - Connecticut Children's

Dr. Brian Cole

Dr. Brian Waterman

Crystal Clinic Orthopedic Center (partnership with Summa Health System)

Dr. Geoffrey Abrams

Dr. Richard F. Howard

Dr. Steven Levin

Ellis and Badenhausen Orthopaedics

Gundersen Health System

Highland Clinic Orthopaedics and Sports Medicine

OrthoIndy - Bone, Joint, Spine, Muscle

Jackson Orthopedic Specialists

Trinity Clinic Orthopedic and Sports Medicine

Keller Orthopedics

Miami Institute for Joint Reconstruction

Mountain Orthopaedics

Raleigh Orthopaedic Clinic

Ortho Illinois

Ortho Carolina

Ortho Virginia

South Shore Hospital

Southeast Georgia Health System

St. Elizabeth Medical Center

Tallgrass Orthopedic and Sports Medicine

Western Orthopaedics 